\title{
Detecting and Minimizing Bad Posture using Postuino Among Engineering Students
}

\author{
Reem Alattas and Khaled Elleithy \\ Department of Computer Science \& Engineering \\ University of Bridgeport \\ Bridgeport, CT, USA \\ ralataas@my.bridgeport.edu, elleithy@bridgeport.edu
}

\begin{abstract}
To understand how good posture minimizes computer related injury's pain, we developed Postuino. A device that warns the computer users if they are leaning too close towards computer screen. Postuino has an accompanying web application that visualizes the collected data and displays a chart to simplify comparing between straight time and slouch time. Also, the app suggests taking frequent breaks to minimize the risk of injuries and to increase productivity. Then, we designed an experiment with different factors to evaluate the efficiency of Postuino. In our study, 24 subjects first use the computer for 3 hours after disabling Postuino's alert system. Afterwards, they use the computer again for two more 3 hour phases after enabling the alert system. We collected data, analyzed it, and presented the results in this paper.
\end{abstract}

Keywords - embedded systems, microcontrollers, Arduino, ultrasonic sensors, posture, experimentation.

\section{INTRODUCTION}

Computers are considered as an essential part of our daily life. Nowadays, computers are used in different environment; including home, school, and work. Although computers made our lives easier and more efficient, there were some injuries related to computer use; such as muscle and joint pain. Using a computer for long periods often results in postural compromises such as kyphosis, i.e. rounding of the upper back, short hip flexors and quadriceps, and forward head position. These in turn lead to head, neck and shoulder tension and pain, lower back pain, improper breathing patterns, nerve compression, and increased potential for disc herniation and arthritis [1]. These problems are caused by bad posture and made worse by sitting for long periods of time. Therefore, we developed Postuino to detect bad posture among computer users and suggest short breaks after sitting and using the computer for a certain amount of time.

A survey of 1,544 graduating seniors at Harvard University, reported that over $50 \%$ of the students experienced symptoms with computer use, and $12.6 \%$ experienced symptoms after computing for 1 hour or less [2]. Risk factors were academic concentration in computer science, female gender, and using a computer more than 20 hour/week. Compared to undergraduate students, graduate students were at greater risk for musculoskeletal symptoms and disorders due to the intensive computer use required for data analysis and thesis writing, as well as employment as graduate student researchers and teaching assistants [3]. Another survey of 304 engineering graduate students at Harvard University reported that $65 \%$ of those students experienced persistent pain in upper extremity or neck during graduate school. Of all participants, $60 \%$ reported recurrent pain was related to computer use.

As computer adoption increases we can expect a corresponding increase in the occurrence of neck pain if appropriate countermeasures are not employed. Thus, we developed Postuino to detect bad posture by measuring the distance between the user and computer monitor in order to indicate if the user is leaning too close to the computer monitor. Postuino alerts the user to prevent slouching and suggests short breaks to minimize the impact of too much sitting and staring at the computer.

Although sitting requires less muscular effort than standing, it still causes physical fatigue because it holds parts of human body steady for long periods of time. This reduces circulation of blood to muscles, bones, tendons and ligaments; which leads to stiffness and pain [4].

In this paper, we propose Postuino, a novel solution for fixing bad posture and reducing muscle pain consequently. Section 2 discusses previous work done in the field of posture detection. Section 3 describes the new tool, its different parts, and the accompanying web application. Then, an experiment is discussed and analyzed in section 4. Finally, section 5 evaluates the new tool and concludes the paper.

\section{RELATED WORK}

Bad posture detection is a field where extensive development has been done. Many devices were developed to detect and correct poor posture such as; iPosture, BASN, and Lumo Back. However, all these devices belong to wearable technology arena. Some of those devices will be discussed next.

iPosture is a small 1-inch round device designed to be worn by the user, detect bad posture, and notify the wearer with a vibration. It warns the user when detecting any deviation greater than three degrees from the chosen posture that lasts longer than one minute. The chosen posture is saved by pushing a button [5]. It is convenient to have such a small posture detection device, though it is uncomfortable to wear all the time. The chosen posture as well might not be the best posture to be kept all the time. This device does not give any feedback to the user about the posture performance during the time where that tool was used.

Body Area Sensor Network (BASN) is a solution for wireless and wearable posture recognition based on a custom-designed wireless body area sensor network, called WiMoCA. Nodes of the network, mounted on different parts 
of the human body, exploit tri-axial accelerometers to detect body postures [6]. The disadvantages of that solution lies in the impracticality of wearing many sensors and purring daily life activities such as working or studying and the lack of feedback provided for users. On the other hand, one of the challenges this tool faces is power consumption optimization.

Lumo Back is a comprehensive bio-mechanical posture sensor and body movement feedback system that monitors the wearer's posture and coaches to improve throughout the day. It is considered one of the best available solutions, because it is only $8.5 \mathrm{~mm}$ thick. The sensor gently vibrates when the user slouches, which is similar to iPosture alert methodology. When synced with a compatible mobile device, Lumo Back keeps track of the user's daily activities; such as steps taken, time spent sitting, calories burned, and sleep habits [7]. The downside of Lumo Back is the lack of a built in adjustor for variations in different users' "correct postures," since each person has a different curvature to their spine, their "correct posture" may be different than someone else's.

All the above devices are worn by the user and target general population without focusing on the reasons that caused bad posture. Postuino aims to detect bad posture for computer users while using their computers. It is the only posture detector device that is not worn. Instead, it can be positioned next to the computer monitor or mounted on top of the monitor depending on the users' preferences. Postuino provides valuable feedback to the users and suggests short breaks to avoid sitting for too long as we will see in the next sections.

\section{POSTUINO}

Postuino warns computer users if they are leaning too close towards the computer screen, by measuring the distance between the user and computer monitor in order to indicate if the user is leaning too close towards the computer monitor. Also, it provides excellent feedback for users about the time they spend sitting and slouching. This information can be used by the users themselves or their physicians or chiropractors for health monitoring purposes. In the next subsections, we will elaborate on the hardware used to make the prototype, distance measurement algorithm, data visualization tool used to convert data to graphical representation, and the accompanying web application to display the visualized data for each user.

\section{A. Hardware}

Our design uses Arduino Micro and ultrasonic sensor. It can be placed next to the computer facing the user, as shown in Fig. 1, or mounted on top of the computer monitor, as shown in Fig. 2. When the user leans towards the computer monitor, the distance between him/her, the computer, and the Postuino accordingly falls below a certain threshold. Then, an LED lights up and Piezo speaker plays chosen melody in order to alert the user to correct his/her posture.

We chose to use Arduino Micro because of its small size compared to other Arduino boards. Moreover, Arduino Micro has pins that can be connected straight onto a prototyping breadboard, which allows easy construction of complex circuits without soldering [8].

The ultrasonic sensor functions on the same principles as radar; it transmits a high-frequency signal and, based on the echo, determines the proximity of a specific object. Ultrasonic sensors can measure the distance of an object accurately at a minimum of 2 centimeters and a maximum of 3 meters from the device. Therefore, we utilize ultrasonic sensor to measure the distance between the user and computer monitor. In our judgment, this sensor would be enough to detect what we would consider a "bad posture".

Two indicators were chosen for this design to notify the user of bad posture, LED and Piezo speaker, in order to make sure users get adequate alert to correct their bad posture.

We did not provide an external battery, because connecting Postuino to a USB cable is very convenient, since the device is meant to correct bad posture of computer users. Fig. 3 shows the circuit design of Postuino and Fig. 4 shows the schematic for that circuit.

\section{B. Distance Measurement}

According to the United States Department of Labor, the preferred viewing distance is between 20 and 40 inches (50 and 100 centimeters) from the eye to the front surface of the computer screen, as shown in Fig. 5. To detect a bad posture, we made the LED and Piezo speaker react when the distance to the user falls below 20 inches (50 centimeters).

As sound travels at 1,130 feet per second, there are 73.746 micro-seconds per inch. This gives the distance travelled by the ultrasonic sensor, outbound, and return, so to find the distance of the user we take half of the distance traveled.

$$
\text { distance in inches }=(\text { duration } / 74) / 2
$$

For metric system users, we use the following equation to calculate the distance in centimeters, since the speed of sound is 29 microseconds per centimeter.

$$
\text { distance in centimeters }=(\text { duration } / 29) / 2
$$

When the user leans too close to the computer screen, the distance between the user, computer, and Postuino falls bellows the defined threshold distance which triggers the LED to light up and the speaker to play the melody. Once the user corrects the posture, the distance goes beyond the threshold which causes the LED to turn off and the speaker to stop playing melody. The reason for this is because our vision of "good posture" involves having the minimal distance between the user and the computer, which implies sitting straight without slouching.

\section{Data Visualization}

Postuino measures the time a user spends sitting and using a computer. The measured time is divided into straight time and slouch time. Since human brains process visual information efficiently, we visualized the collected data using Plotly.

Plotly is a platform for analyzing and visualizing data streaming from any hardware device. Arduino API allows continuously transmitting data or transmitting a single chunk of data from Arduino and then making interactive graphs in the browser. 
We used Arduino StopWatch library to measure elapsed time. Also, we used Plotly Arduino Ethernet Library to graph the data. The graphs are embedded in Postuino's web application that will be described next.

\section{Web Application}

The accompanying web application was developed using Bootstrap to allow easy switching between mobile, tablet, and desktop views. Each user can sign up to create a new account. Then, sign in to retrieve live feedback about the time spent sitting either straight or slouching to use the computer. The application also sends push notifications to users who spent a certain amount sitting in order to take a short break and purse working after the break. The default time threshold is 20 minutes and it can be changed by the user at any time using the web application.

The web application displays a two dimensional stacked area chart for the straight sitting time and the slouching time for the current day, five days, and one month. This chart can be shared in social media channels or emailed to the user's physician or chiropractor. It also displays a two dimensional bar chart to simplify the comparison between straight time and slouching time. Fig. 6 displays both charts for a sample user.

\section{EXPERIMENT DESIGN}

In our study, we setup a workstation and mounted Postuino on top of the screen to record the elapsed time, including straight and slouch time. First, $(\mathrm{N}=24)$ subjects used the computer for 3 consecutive hours after disabling the alert system in Postuino in order to record the elapsed time, without alerting the user to correct the bad posture. Then, we repeated the first stage after enabling the alert system in Postuino as follows; alert contains light only, sound only, both light and sound. Finally, the second stage was repeated after re-positioning Postuino to take place next to computer monitor. We collected data with Postuino and a questionnaire.

\section{A. Participants}

The 24 engineering graduate students recruited for the experiment have experienced computer related injury during their graduate study. All of them were required to sit and use a computer for 3 consecutive hours in 3 different stages.

At the beginning of the experiment, all students were asked questions regarding their gender, age, major, and type of computer related injury they have. There were 9 females and 15 males, all of age $24-35$ as Table 1 shows.

Fig. 7 shows the distribution of our sample subjects by major. Since all participants are enrolled in a Master's degree program, Fig. 8 shows the prevalence of computer use by year of graduate study in first and second year. Finally, Fig. 9 shows the prevalence of muscle or joint pain among engineering graduate students due to computer use.

\section{B. Design Rationale}

Several design choices were made during the experiment design. Ultimately, we chose an experiment setup with the following features: disabling Postuino alert system in phase 1 while using it to record elapsed time and enabling Postuino alert system in phase 2 and 3 . In the first part of phase 2 and 3 , the alert system contains light only. In the second part of phase 2 and 3, the alert system contains sound only. In the third and final part of phase 2 and 3, the alert system contains both light and sound. The difference between phase 2 and 3 is the position of Postuino. It is positioned on top of the computer monitor in phase 2 and next to the monitor in phase 3 . There was 30 minutes break between the phases.

Two surveys were given to each participant. The first survey is given to each participant before the experiment and it contains questions about gender, age, major, hours per week of computer use and the type of computer related injury. The results of this survey are shown in Table 1 and Fig. 7, 8, and 9. The second survey has 4 parts with the same questions and a fifth different part. Part 1 will be answered before the experiment; part 2 will be answered after phase 1; part 3 will be answered after phase 2 ; and part 4 will be answered after phase 3 . Each part has a question about the pain level during computing. The pain level is measured using Wong-Baker faces paint rating scale as shown in Fig.10. Finally, the fifth part has questions to review Postuino's efficiency.

\section{Evaluation}

According to our experiment, Postuino produced significant effects in detecting and correcting bad posture. $17 \%$ of the participants felt less pain in phase 2 . Of all participants, $33 \%$ of the participants felt less pain in phase 3 . These numbers reflect the improvement in only one session. Therefore, $87.5 \%$ of the participants agreed that Postuino can improve their posture and $75 \%$ of the participants found the break reminders in the web app are beneficial. Fig. 11, 12, 13 , and 14 show the results of the second survey that was answered by each participant after the experiment.

In regards to Postuino's setup, 67\% of the participants preferred positing Postuino on top of the screen because it gives better visibility. From our point of view, mounting Postuino on top of the screen gave better readings. On the other hand, $71 \%$ preferred light only, so they can use Postuino in a public area such as library or laboratory.

Finally, Fig. 16 compares between straight time and slouch time for a randomly selected user in the three phases of the experiment. The minimum slouch time happened when Postuino was mounted on top of the computer, specifically in the third hour when both light and sound were used as Figure 17 shows.

\section{CONCLUSION}

In our tests of Postuino's efficiency, we have been able to prove that improving the posture helps reducing muscle and joint pain that is related to computer use. From quantitative data, we can recommend the use of Postuino to detect bad posture, minimize slouch time, and suggest frequent breaks that minimizes the risk of getting computer related injuries.

Mounting Postuino on top of the screen gave better readings. Still, users can position it next to the computer monitor depending on their personal preference. Also, enabling light alert only while disabling sound alerts allows 
users to use Postuino in public places for maximum benefit and better productivity. However, combining sound and light alerts yields the best results in minimizing slouch time. In sum, users have the option of choosing the appropriate alert method depending on their needs and their situation.

TABLE I. SAMPLE DEMOGRAHICS

\begin{tabular}{|l|l|}
\hline & $\begin{array}{l}\text { Participants } \\
(\mathbf{N}=\mathbf{2 4 )}\end{array}$ \\
\hline Gender: Male & $62.5 \%(15)$ \\
\hline Age: $24-29$ & $75 \%(18)$ \\
\hline Age: $30-35$ & $25 \%(6)$ \\
\hline
\end{tabular}

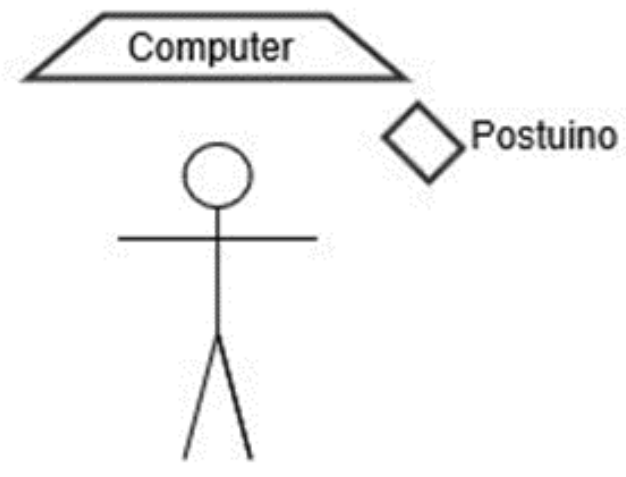

User

Fig. 1. Postuino's Side Position

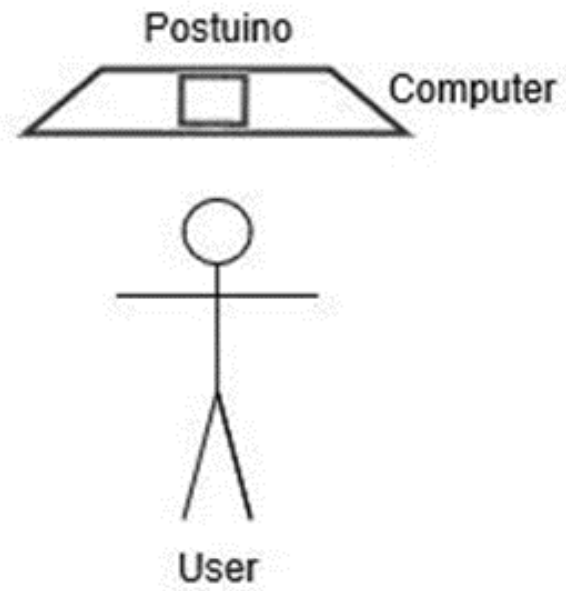

Fig. 2. Postuino's Front Position

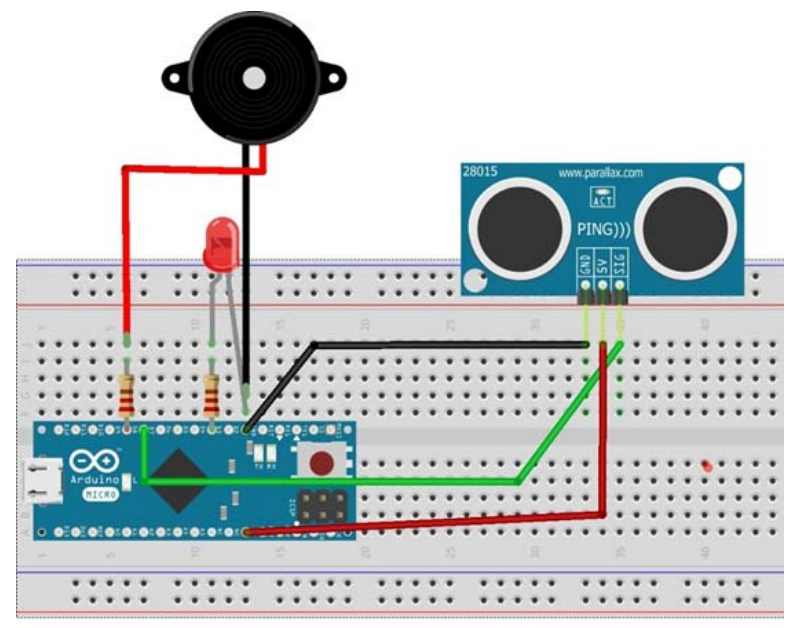

Fig. 3. Postuino Circuit Design

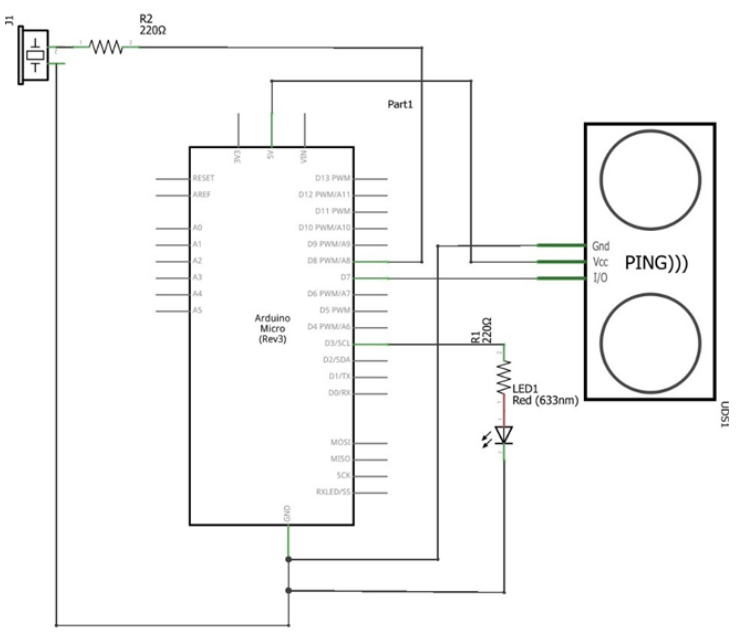

Fig. 4. Postuino Schematic

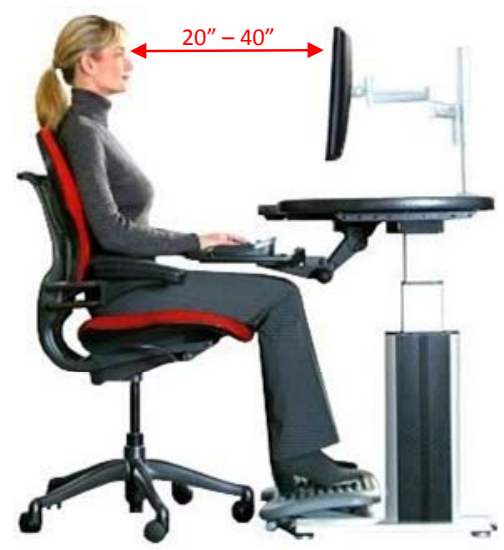

Fig. 5. Preferred Viewing Distance 


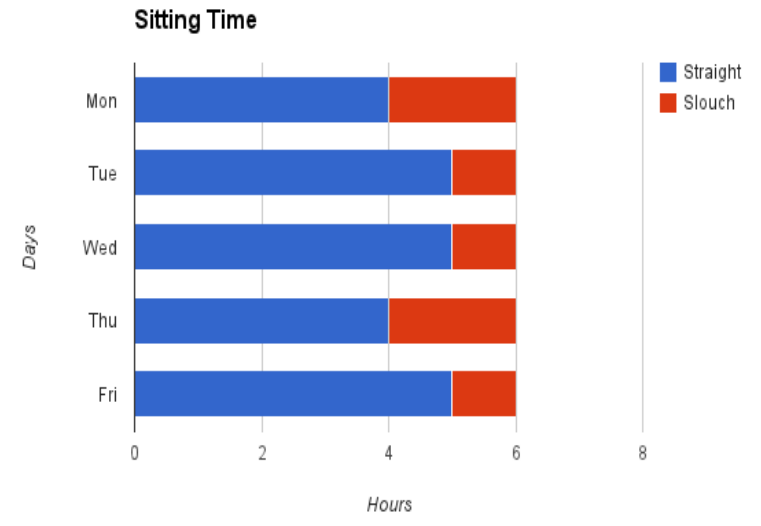

Fig. 6. Sitting Time Chart for a Sample User on the Web App

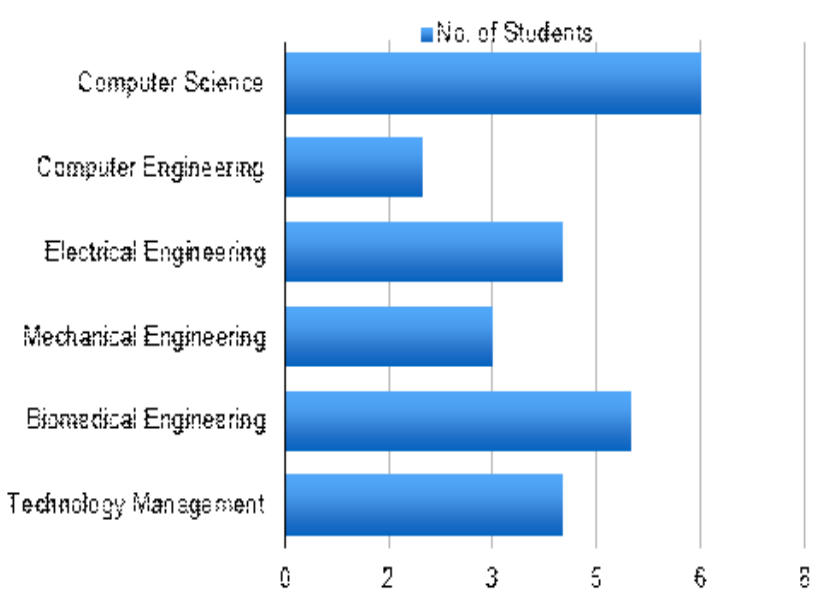

Fig. 7. Number of Students per Engineering Major
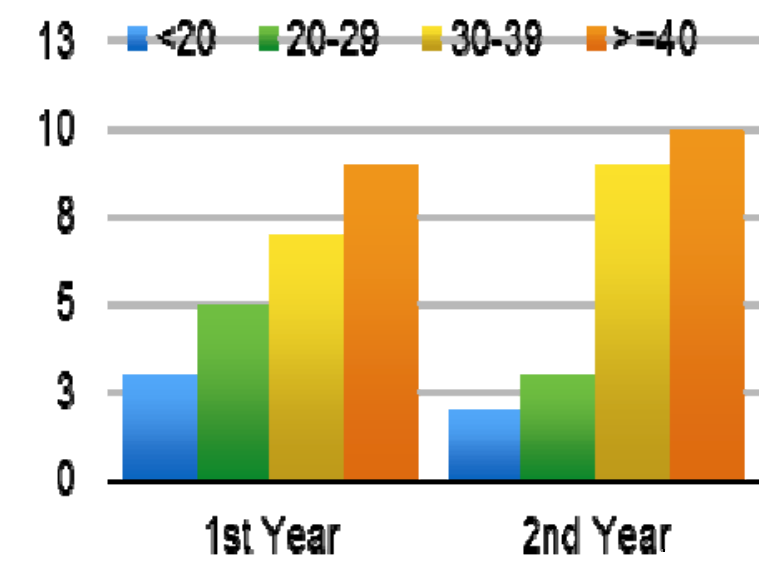

Fig. 8. Hours per week of Computer Use

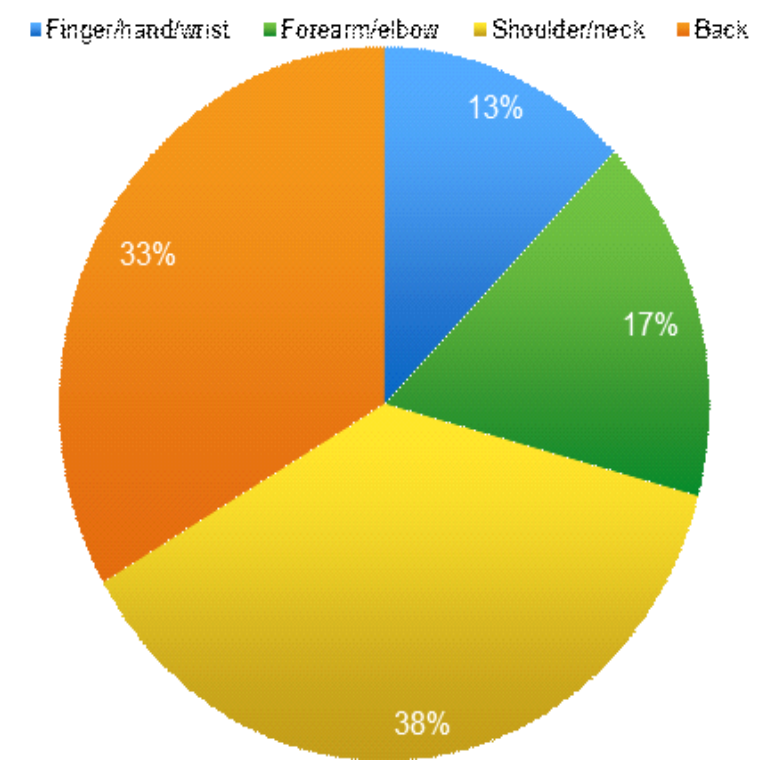

Fig. 9. Prevalence of Computer-related Injurie

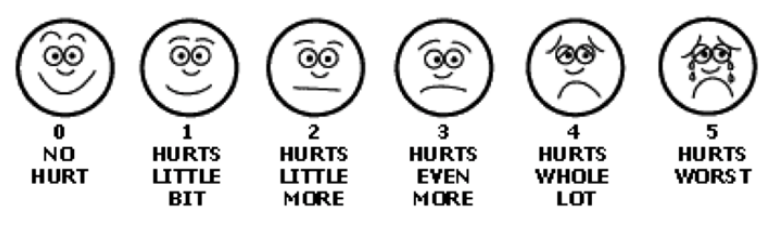

Fig. 10. Wong-Baker Faces Pain Rating Scale

12

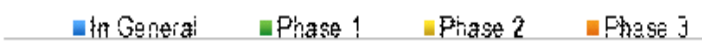

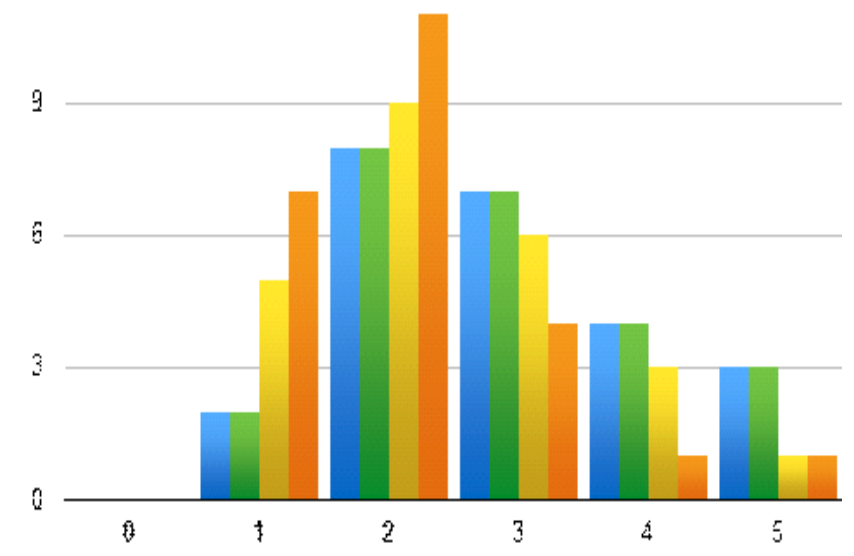

Fig. 11. Pain Level During Computing (In General and During Phase 1, 2, 3) 


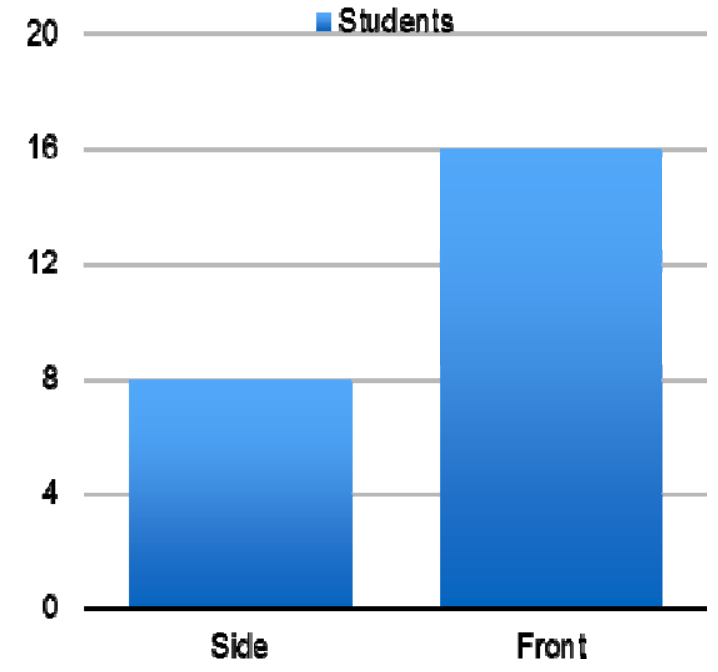

Fig. 12. Postuino's Preferred Position

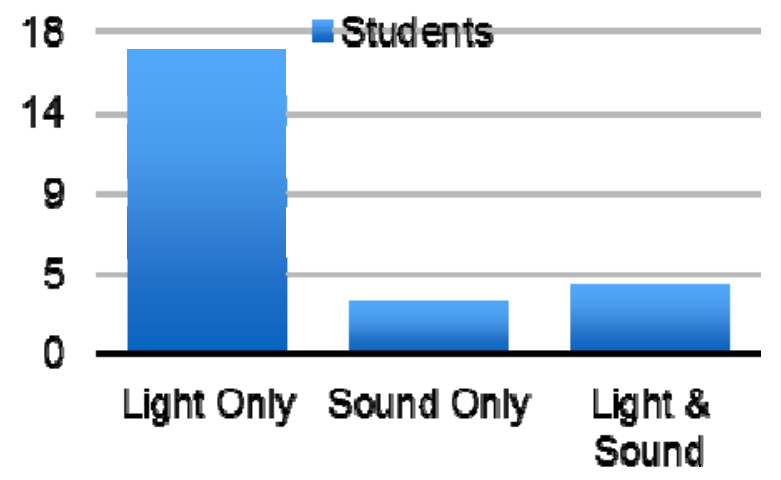

Fig. 13. Postuino's Preferred Alert Method

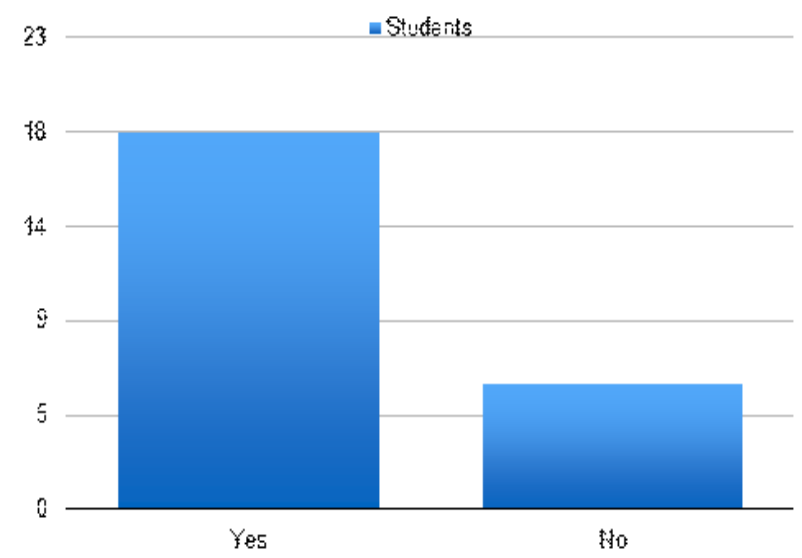

Fig. 14. Did Break Reminders help in improving the situation?

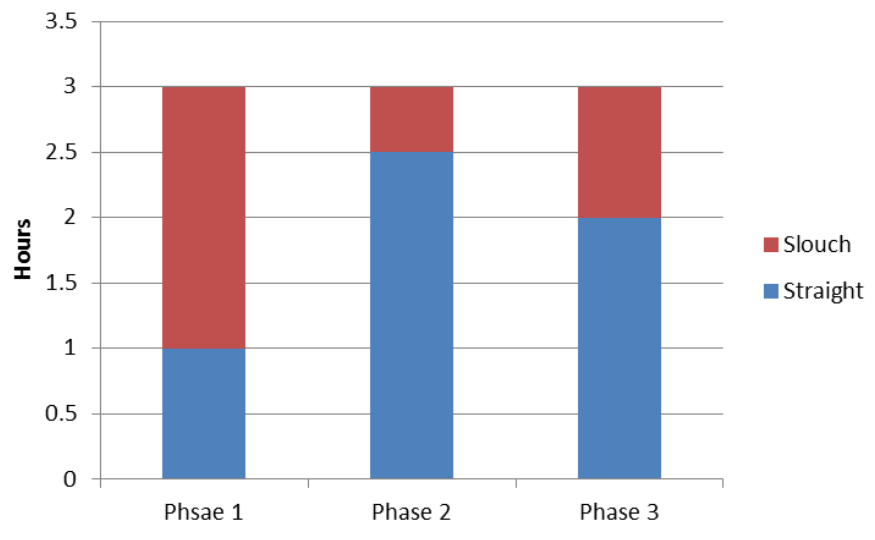

Fig. 15. Sraight Time vs. Slouch Time for a Randomly Selected User in Phase 1,2, and 3

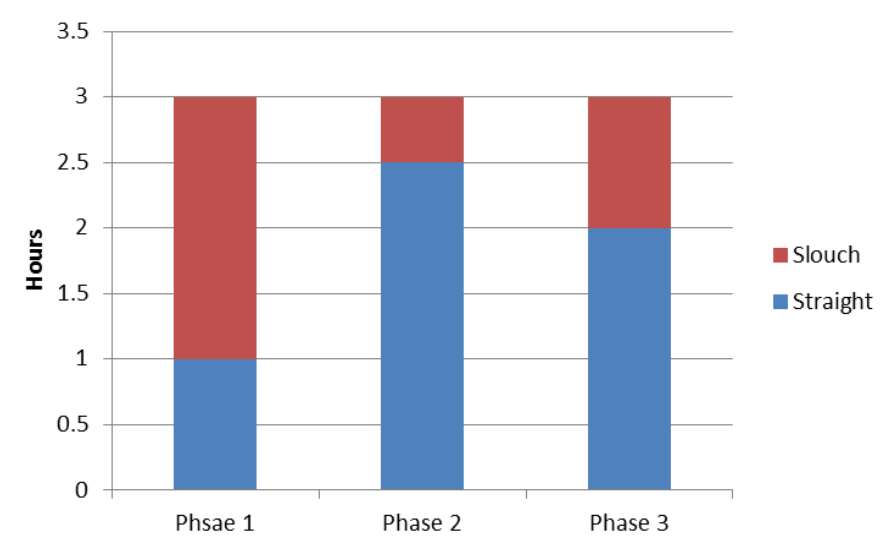

Fig. 16. Sraight Time vs. Slouch Time for a Randomly Selected User in Phase 1,2, and 3

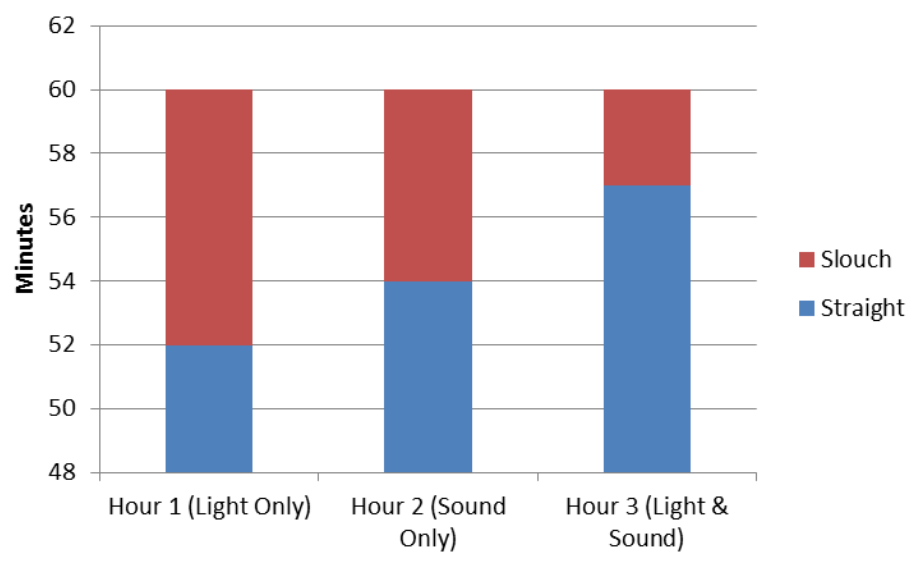

Fig. 17. Phase 2 breakdown ino 3 Hours 


\section{REFERENCES}

[1] Charlotte Ord. 3 every day items that could be stopping you from losing weight. November 7th, 2011. http://charlotteord.blogspot.com/2011/11/3-every-day-items-that-arestopping-you.html

[2] United States Department of Labor Occupational Safety and Health Administration

https:/www.osha.gov/SLTC/etools/computerworkstations/component s_monitors.html

[3] iPosture home page, http://www.iposture.com/

[4] Farella, E., Pieracci, A., Benini, L., \& Acquaviva, A. (2006, June). A wireless body area sensor network for posture detection. In
Computers and Communications, 2006. ISCC'06. Proceedings. 11th IEEE Symposium on (pp. 454-459).

[5] Computer-related injuries (2014), www.betterhealth.vic.gov.au.

[6] Leah Buechley’s LilyPad Arduino. http://web.media.mit.edu/ leah/ LilyPad/index.html.

[7] Leah Buechley's turn signal jacket, http://web.media.mit.edu/ /eah/ LilyPad/build/turn_signal_jacket.html.

[8] Robbins, M., Johnson, I. P., \& Cunliffe, C. (2009). Encouraging good posture in school children using computers. Clinical Chiropractic, 12(1), 35-44. 